generally saw a stronger effect for squamous- and small cell lung carcinomas than for adenocarcinomas. Smoking and simultaneous exposure to other occupational exposures exerted a minor confounding effect on the risk estimates. The effect modifications with smoking tended to be supra-additive.

Conclusions SYNERGY adds valuable knowledge to the field of occupational cancer epidemiology, and underlines the importance to collect data on histology, and lifelong information on occupational exposures and smoking.

\section{EXPOSURE-RESPONSE ESTIMATES FOR DIESEL ENGINE EXHAUST AND LUNG CANCER MORTALITY BASED ON DATA FROM THREE OCCUPATIONAL COHORTS}

${ }^{1}$ Roel Vermeulen, ${ }^{2}$ Debra Silverman, ${ }^{3}$ Eric Garshick, ' 'Jelle Vlaanderen, 'Lützen Portengen, ${ }^{4}$ Kyle Steenland. 'IRAS Utrecht University, Utrecht, The Netherlands; ${ }^{2}$ National Cancer Institute, Bethesda, MD, USA; ${ }^{3}$ Harvard Medical School, Boston, MA, USA; ${ }^{4}$ Emory University, Atlanta, GA, USA

\subsection{6/oemed-2014-102362.145}

Objectives To derive a meta-exposure-response curve (ERC) for DEE and lung cancer mortality and estimate lifetime excess risks (ELRs) of lung cancer mortality based on assumed occupational and environmental exposure scenarios.

Method We conducted a meta-regression of lung cancer mortality and cumulative exposure to elemental carbon (EC), a proxy measure of DEE, based on relative risk (RR) estimates reported by three large occupational cohort studies. Based on the derived risk function, we calculated ELRs for several lifetime occupational and environmental exposure scenarios, and also calculated the fractions of annual lung cancer deaths attributable to DEE.

Results We estimated a lnRR of 0.00098 (95\% CI: 0.00055 , $0.0014)$ for lung cancer mortality with each $1-\mu \mathrm{g} / \mathrm{m}$ 3-year increase in cumulative EC. Estimated numbers of excess lung cancer deaths through age 80 for lifetime occupational exposures of 1,10 , and $25 \mu \mathrm{g} / \mathrm{m} 3 \mathrm{EC}$ were 17, 200, and 689 per 10000 , respectively. For lifetime environmental exposure to $0.8 \mu \mathrm{g} / \mathrm{m} 3$ EC, we estimated 21 excess lung cancer deaths per 10000 . Based on broad assumptions regarding past exposures we estimate that approximately $6 \%$ of annual lung cancer deaths may be due to DEE exposure.

Conclusions Combined data from three US occupational cohort studies suggest that DEE at levels common in the workplace and in outdoor air appear to pose substantial excess lifetime risks of lung cancer, above usually acceptable limits in the US and Europe, which are generally set at 1/1000 and 1/100 000 based on lifetime exposure for the occupational and general population, respectively.

\section{MORTALITY OF A COHORT OF WORKERS IN GREAT BRITAIN WITH BLOOD LEAD MEASUREMENTS}

${ }^{1}$ Damien McElvenny, ${ }^{1}$ Brian Miller, ${ }^{1}$ Laura MacCalman, 'Anne Sleeuwenhoek, ${ }^{1}$ Martie van Tongeren, ${ }^{2}$ Kevin Shepherd, ${ }^{2}$ Andrew Darnton, ${ }^{1}$ John Cherrie. ${ }^{1}$ Institute of Occupational Medicine, Edinburgh, UK; ${ }^{2}$ Health and Safety Executive, Bootle, UK

\subsection{6/oemed-2014-102362.146}

Objectives We report on the first ever analysis of a UK cohort of workers with blood lead level measurements that was assembled in the late 1970s.
Method As an alternative to mean and maximum blood lead levels, we carried out an exposure assessment that assigned workers to high, medium or low exposure to lead. We additionally assessed whether workers would be exposed to an important level of relevant co-carcinogens.

Results 3466 deaths were observed among 7770 men and 1352 women. The SMRs for all causes $(109,95 \%$ CI 105-112) and all malignant neoplasms (113,107-120) were significantly raised. SMRs for oesophageal, stomach, bladder, brain and kidney cancer and non-malignant kidney disease were not raised, but were raised for lung cancer (142,129-157). The SMR for circulatory diseases $(105,99-100)$ was of borderline significance. No trends were observed for mean or maximum blood lead level or assessed lead exposure for any of the cancers of a priori interest, but a significant association was found for circulatory diseases (ischaemic heart disease) with mean and maximum blood lead level.

Conclusions The excess of lung cancer is possibly to be due to tobacco smoking. This study provides strong evidence to support an association between increased lead exposure and increased risk of ischaemic heart disease mortality. The study is, however, limited by the lack of complete occupational histories for the included participants.

\section{OCCUPATIONAL EXPOSURE TO CARCINOGENS IN NEW ZEALAND}

Andrea't Mannetje, David McLean, Bill Glass, Kerry Cheung, Jeroen Douwes, Neil Pearce. Centre for Public Health Research, Massey University, Wellington, New Zealand

\subsection{6/oemed-2014-102362.147}

Objectives To provide a detailed assessment of the extent and industrial distribution of workplace carcinogens in New Zealand, and to identify key industries and key carcinogens for which intervention would result in marked reductions in occupational cancer.

Method A literature review was conducted identifying the carcinogens present in and the cancer risks associated with each respective industry. A New Zealand specific Information System on Occupational Exposure to Carcinogens (NZ-CAREX) was developed.

Results There are more than 50 known human carcinogens commonly present in New Zealand workplaces. The most common of these are: asbestos; benzene; chromium VI compounds; formaldehyde; involuntary smoking; occupational exposures as a painter; silica; solar radiation; wood dust. The industries for which an increased cancer risk has been observed repeatedly in epidemiological studies and have the highest number of potentially exposed workers include: agriculture; construction; health services; machinery and equipment manufacturing; metal product manufacturing; and wood/paper product manufacturing. Of the agricultural chemicals currently in use in New Zealand's agricultural sector, none are known human carcinogens, but numerous are suspected to be carcinogenic to humans. A number of barriers for the implementation of intervention strategies were identified, which could be removed by improving access to knowledge regarding the occurrence of carcinogens in New Zealand workplaces and improving access to control tools and methods to evaluate their effectiveness.

Conclusions Exposure to carcinogens remains common in New Zealand workplaces, and many opportunities remain for reducing exposure and the associated occupational cancer burden. 